\title{
Grape seed procyanidin B2 ameliorates mitochondrial dysfunction and inhibits apoptosis via the AMP-activated protein kinase-silent mating type information regulation 2 homologue 1-PPAR $\gamma$ co-activator- $1 \alpha$ axis in rat mesangial cells under high-dose glucosamine
}

\author{
Lei Baot, Xiaxia Cait, Zhaofeng Zhang* and Yong Li* \\ Department of Nutrition and Food Hygiene, School of Public Health, Peking University, Beijing 100191, \\ People's Republic of China \\ (Submitted 7 April 2014 - Final revision received 12 September 2014 - Accepted 2 October 2014 - First published online 18 November 2014)
}

\section{Abstract}

Grape seed procyanidin B2 (GSPB2), an antioxidative and anti-inflammatory polyphenol in grape seed, has been found to have protective effects on diabetic nephropathy. Based on its favourable biological activities, in the present study, we aimed to investigate whether GSPB2 could inhibit apoptosis in rat mesangial cells treated with glucosamine (GlcN) under high-dose conditions. The results showed that the administration of GSPB2 $(10 \mu \mathrm{g} / \mathrm{ml})$ significantly increased the viability of mesangial cells treated with GlcN at a dose of $15 \mathrm{~mm}$. We found that GSPB2 inhibited apoptosis in mesangial cells using terminal deoxynucleotidyl transferase-mediated deoxyuridine triphosphates (dUTP) nick-end labelling staining and flow cytometry technique ( $P<0.05$ for both). GSPB2 treatment also suppressed oxidative stress by elevating the activity of glutathione peroxidase $(P<0.05)$ and superoxide dismutase $(P<0.01)$, as well as prevented cellular damage. GSPB2 enhanced the mRNA expression of nuclear respiratory factor 1 , mitochondrial transcription factor A and mitochondrial DNA copy number in mesangial cells as determined by real-time PCR ( $P<0.05$ for each). Finally, GSPB2 treatment activated the protein expression of PPAR $\gamma$ co-activator- $1 \alpha$ (PGC-1 $\alpha$ ), silent mating type information regulation 2 homologue 1 (SIRT1) and AMP-activated protein kinase (AMPK) in mesangial cells, These findings suggest that GSPB2 markedly ameliorates mitochondrial dysfunction and inhibits apoptosis in rat mesangial cells treated with high-dose GlcN. This protective effect could be, at least in part, due to the activation of the AMPK-SIRT1-PGC-1 $\alpha$ axis.

Key words: Apoptosis: Diabetic nephropathy: Grape seed procyanidin B2: Mesangial cells: Mitochondrial dysfunction

Diabetic nephropathy (DN) is one of the most serious and common microvascular complications of diabetes mellitus, and is a major cause of end-stage renal disease ${ }^{(1)}$. Mesangial cells play a vital role in the pathogenesis of DN. Mesangial cell proliferation and hypertrophy have been identified to be early events in the progress of diabetic kidney disease ${ }^{(2)}$. However, mesangial cell apoptosis, which has been observed in both experimental and human glomerular diseases including DN, becomes an important event as the disease progresses $^{(3-5)}$. It has been demonstrated that mesangial cell apoptosis is related to an increase in albuminuria ${ }^{(6)}$, and may play a pathological role in progressive glomerulosclerosis by reducing the number of mesangial cells ${ }^{(4)}$. Therefore, seeking effective methods for inhibiting mesangial cell apoptosis may be of great clinical importance in the treatment of DN.

Oxidative stress is considered to be a trigger of mesangial cell apoptosis ${ }^{(7)}$. Reactive oxygen species (ROS), such as $\mathrm{O}_{2}^{-\cdot}, \mathrm{H}_{2} \mathrm{O}_{2}, \mathrm{HO}_{2}^{-}$and ${ }^{\circ} \mathrm{OH}$, are generated when stimulated by pro-inflammatory factors. Excess ROS generation leads to increased metabolite accumulation in diabetes ${ }^{(8,9)}$. There is mounting evidence that ROS initiate mesangial cell apoptosis in vitro ${ }^{(10,11)}$. Mitochondria are the major source of ROS. Mitochondrial dysfunction could lead to more ROS generation and result in further oxidative stress and increased apoptosis.

Abbreviations: AMPK, AMP-activated protein kinase; DN, diabetic nephropathy; FBS, fetal bovine serum; GAPDH, glyceraldehyde 3-phosphate dehydrogenase; GlcN, glucosamine; GSH-Px, glutathione peroxidase; GSPB2, grape seed procyanidin B2; MDA, malondialdehyde; MEM, minimum essential medium; mtDNA, mitochondrial DNA; MTT, 3-(4,5-dimethylthiazol-2-yl)-2,5-diphenyltetrazolium bromide; NRF-1, nuclear respiratory factor 1; PARP, poly(ADP-ribose) polymerase; PGC- $1 \alpha$, PPAR $\gamma$ co-activator $1 \alpha$; RAGE, receptor for advanced glycation end products; ROS, reactive oxygen species; SIRT1, silent mating type information regulation 2 homologue 1; SOD, superoxide dismutase; TFAM, mitochondrial transcription factor A; TUNEL, terminal deoxynucleotidyl transferase-mediated dUTP nick-end labelling.

*Corresponding authors: Z. Zhang, email zhangzhaofeng@126.com; Y. Li, fax +86 10 82801177, email liyongbmu@163.com

† Both authors contributed equally to this work. 
It has been reported that mitochondrial dysfunction resulting from the decrease in mitochondrial DNA (mtDNA) is associated with the pathogenesis of diabetes mellitus and its complications including $\mathrm{DN}^{(12)}$. Therefore, the inhibition of mitochondrial dysfunction may be an effective way to suppress apoptosis and slow down the progression of DN.

PPAR $\gamma \quad$ CO-activator- $1 \alpha$ (PGC- $1 \alpha)$, a transcriptional CO-activator, is highly expressed in tissues with high-capacity mitochondrial systems such as brown adipose tissue, skeletal muscle, heart and kidneys ${ }^{(13,14)}$. The overexpression of the transcriptional co-activator PPAR (PGC- $1 \alpha$ ) can prevent aldosterone-induced mitochondrial dysfunction in podocytes and regulate mitochondrial oxidative stress ${ }^{(15,16)}$. AMP-activated protein kinase (AMPK) and silent information regulator T1 (SIRT1) have been found to be capable of regulating PGC- $1 \alpha$ activity and energy metabolism of mitochondria ${ }^{(17)}$. It has been suggested that high glucose-induced mesangial cell damage lowered the activity of the AMPK-SIRT1-PGC- $1 \alpha$ axis and exacerbated oxidative stress as well as apoptotic cell damage in diabetes ${ }^{(18)}$. Therefore, targeting the activation of the AMPK-SIRT1-PGC-1 $\alpha$ axis may offer new approaches for the treatment of mesangial cell apoptosis and DN.

Proanthocyanidin extracts, derived from grape seed, have been reported to possess a wild range of potent biological activities including free radical scavenging, antioxidant, anti-inflammatory and anti-tumour activities ${ }^{(19,20)}$. Grape seed procyanidin B2 (GSPB2) is one of the main components of proanthocyanidin extracts from grape seed, and accounts for $6.4 \%$ in grape seed ${ }^{(21)}$. GSPB2 is composed of two molecules of flavan-3-ol (-)-epicatechin linked by a $4 \mathrm{~b} \rightarrow 8$ bond. It has more powerful bioactivity than other water-soluble polyphenols. Previous studies have found that GSPB2 could prevent ROS generation, inhibit endothelial cell apoptosis, and has protective effects against $\mathrm{DN}^{(22,23)}$. However, most of the studies investigating the effects of GSPB2 on DN have focused on animal models, and there is little information available about the benefits of GSPB2 on DN in in vitro systems.

The aim of the present study was to investigate the potential protective effect of GSPB2 on apoptosis and mitochondrial dysfunction in rat mesangial cells treated with high-dose glucosamine $(\mathrm{GlcN})$, and to explore whether this protective effect was, at least in part, due to the activation of the AMPK-SIRT1-PGC- $1 \alpha$ axis

\section{Materials and methods}

\section{Chemicals and reagents}

GSPB2 was purchased from Jianfeng Inc. (>95\% pure, batch no. 20080915). Minimum essential medium (MEM) and fetal bovine serum (FBS) were obtained from Gibco Invitrogen Corporation. Trypsin, dimethyl sulfoxide (DMSO), GlcN and 3-(4,5-dimethylthiazol-2-yl)-2,5-diphenyltetrazolium bromide (MTT) were all purchased from Sigma. The terminal deoxynucleotidyl transferase-mediated deoxyuridine triphosphates (dUTP) nick-end labelling (TUNEL) detection kit was obtained from KeyGen Biotech. The Annexin-V-fluorescein (FLUOS) Staining Kit was obtained from Roche Diagnostics.

\section{Cell culture}

Rat mesangial cells (HBZY-1) were obtained from the Center of Type Culture Collection, Chinese Academy of Medical Sciences. The cells were cultured in MEM supplemented with $10 \% \mathrm{FBS}$ and incubated at $37^{\circ} \mathrm{C}$ under a humidified atmosphere of $5 \% \mathrm{CO}_{2}$. The cells were used at the fifth passage. All experiments were performed in the logarithmic phase of cell growth. The cells were incubated for $24 \mathrm{~h}$ in serum-deprived $(0 \cdot 2 \%$ FBS $)$ MEM. Then, mesangial cells were cultured in MEM and 10\% FBS with or without $15 \mathrm{~mm}-$ GlcN and two concentrations of GSPB2 $(2.5$ and $10 \mu \mathrm{g} / \mathrm{ml})$ for $24 \mathrm{~h}$. The highest concentration of GSPB2 in plasma was $14 \mu \mathrm{g} / \mathrm{ml}$ after being taken for $2 \mathrm{~h}$, so these two concentrations of GSPB2 can be reached in vivo. Cells treated with $15 \mathrm{~mm}-$ mannitol instead of GlcN were used as osmolarity control.

\section{3-(4,5-Dimethylthiazol-2-yl)-2,5-diphenyltetrazolium bromide assay}

MTT assay was used to measure cell viability. Mesangial cells $\left(10^{4}\right.$ cells/well) were incubated in ninety-six-well plates. MEM was removed and replaced with serum-deprived ( $0 \cdot 2 \%$ FBS) medium, and incubated for $24 \mathrm{~h}$ before the experiment started. The cells were then treated with high-dose GlcN (15 mm) with or without the two concentrations of GSPB2 $(2.5$ and $10 \mu \mathrm{g} / \mathrm{ml})$ or mannitol $(15 \mathrm{~mm})$ for another $24 \mathrm{~h}$. Then, $100 \mu \mathrm{l}$ MTT $(0.5 \mathrm{mg} / \mathrm{ml})$ were added. After continued incubation at $37^{\circ} \mathrm{C}$ for an additional $4 \mathrm{~h}$, the medium was carefully removed. The cells were dissolved in $100 \mu \mathrm{l}$ DMSO in each well, and the optical density of eight wells for each group was read at $570 \mathrm{~nm}$ using a microplate reader. Values were normalised to those of cells in the control group, which were considered $100 \%$ viable.

\section{Terminal deoxynucleotidyl transferase-mediated dUTP nick-end labelling assays and flow cytometry}

Apoptosis analysis was performed by TUNEL and flow cytometry. Mesangial cells were seeded into six-well plates at a density of $1 \times 10^{5}$ cells $/ \mathrm{ml}$. Then, the cells were subjected to TUNEL assays according to the manufacturer's protocol. After various treatments, the cells were fixed in $4 \%$ paraformaldehyde for $30 \mathrm{~min}$ under ambient temperature. Then, the cells were washed three times with PBS and permeabilised with $1 \%$ Triton X-100 for 3-5 min. After being washed with PBS and blocked with $3 \% \mathrm{H}_{2} \mathrm{O}_{2}$, the samples were incubated with $50 \mu \mathrm{l}$ of terminal deoxynucleotidyl transferase enzyme reaction solution $(45 \mu \mathrm{l}$ equilibration buffer, $1 \cdot 0 \mu$ l tetramethyl rhodamine isothiocyanate (TRITC)-5-dUTP and $4.0 \mu$ l terminal deoxynucleotidyl transferase enzyme) for $1 \mathrm{~h}$ at $37^{\circ} \mathrm{C}$, and then analysed at a detection wavelength of $573 \mathrm{~nm}$ and an excitation wavelength of $543 \mathrm{~nm}$ by fluorescence microscopy (Nikon Eclipse TE2000-S).

For flow cytometry, we used an Annexin V-fluorescein isothiocyanate (FITC) and propidium iodide double-staining kit (Roche Diagnostics), following the manufacturer's instructions. Briefly, $10^{6}$ cells were collected after being trypsinised, washed with cold PBS, and then centrifuged at $200 \boldsymbol{g}$ for $5 \mathrm{~min}$, and the cells were resuspended in $100 \mu \mathrm{l}$ of Annexin-V-FLUOS and 
propidium iodide mixture, and incubated for $15 \mathrm{~min}$ at $25^{\circ} \mathrm{C}$ in the dark. The samples were analysed by flow cytometry (BD FACSCalibur).

\section{Colorimetric method}

The colorimetric method was applied to detect the levels of glutathione peroxidase (GSH-Px), superoxide dismutase (SOD) and malondialdehyde (MDA), which were used to reflect the severity of oxidative stress. Mesangial cells $\left(1 \times 10^{7}\right.$ in $15 \mathrm{ml}$ medium) were seeded in $75 \mathrm{~cm}^{2}$ tissue culture flasks. The results were calculated with the standard curve, according to the manufacturer's instructions (Beyotime Biotechnology).

\section{Quantitative real-time PCR and analyses of mitochondrial DNA content}

Total RNA and DNA from $10^{6}$ mesangial cells were isolated by TRIzol reagent (Invitrogen) and the DNeasy Tissue Kit (Qiagen Sciences), respectively. The sequences are shown in Table 1 . The expression level of target genes, nuclear respiratory factor $1(N R F-1)$ and mitochondrial transcription factor $\mathrm{A}$ (TFAM), was determined by real-time RT-PCR, and the relative mtDNA content was detected using quantitative real-time PCR. Reverse transcription was performed using a reaction kit according to the manufacture's protocol (Promega Reverse Transcriptase System). The ABI 7300 Real-time PCR Detection System was used to perform real-time PCR. mtDNA and $\beta$-actin were used for the amplification of nuclear and mtDNA, respectively. Cycling conditions were as follows: denaturation programme $\left(95^{\circ} \mathrm{C}\right.$ for $\left.10 \mathrm{~min}\right)$, and amplification and quantification by forty cycles of $95^{\circ} \mathrm{C}$ for $15 \mathrm{~s}$ and $60^{\circ} \mathrm{C}$ for $30 \mathrm{~s}$. The values were determined relative to the control sample after normalising to the values of $\beta$-actin as the control gene, and calculated by the comparative cycle threshold $\left(\Delta \Delta C_{\mathrm{t}}\right)$ method.

\section{Western blot analysis}

Cells from each group were collected and lysed for Western blotting. The whole process of operation was completed on ice. Protein concentrations were determined using a BCA protein assay kit (Beyotime Biotechnology), following the manufacturer's instructions. Equal amounts of protein were separated by $10 \%$ SDS-PAGE and transferred to polyvinylidene fluoride membranes. After being blocked with $5 \%$ non-fat milk or $5 \%$ bovine serum albumin for $1 \mathrm{~h}$, the membranes were incubated overnight at $4^{\circ} \mathrm{C}$ with the following primary antibodies: phosphoAMPK Thr ${ }^{172}$ (1:200; Santa Cruz Biotechnology), SIRT1 (1:200; Santa Cruz Biotechnology), PGC-1 $\alpha$ (1:1000; Cell Signaling
Technology), poly(ADP-ribose) polymerase (PARP, 1:1000; Cell Signaling Technology), glyceraldehyde 3-phosphate dehydrogenase (GAPDH, 1:500; ZSGB-BIO), receptor for advanced glycation end products (RAGE, 1:200; Santa Cruz Biotechnology) and $\beta$-actin (1:1000; Cell Signaling Technology). Goat anti-rabbit or anti-mouse IgG (1:4000; ZSGB-BIO) was used as the secondary antibody. Then, immune complexes were detected by enhanced chemiluminescence with Super ECL Plus Detection Reagent (Applygen) and analysed using Image Pro Plus 6.0 Software (Media Cybernetics). Values were corrected with those of $\beta$-actin used as the control.

\section{Statistical analysis}

Data were assessed by SPSS 13.0 (SPSS Inc.). Results are presented as means and standard deviations. Homogeneity of the data was first tested by selecting the option 'Homogeneity of variance' in SPSS software. If variances were equal, data were evaluated by means of a one-way ANOVA with Bonferroni correction; otherwise, Tamhane's T2 test was performed. $P<0.05$ was considered as statistically significant.

\section{Results}

Effects of grape seed procyanidin B2 on the viability of mesangial cells treated with high-dose glucosamine

The number of mesangial cells cultured under normal glucose concentrations was not altered by GSPB2 treatment (Fig. 1(a)). In contrast, GlcN significantly suppressed cell viability compared with the control group $(P<0 \cdot 05)$. However, cell viability was significantly improved in the GSPB2 $(10 \mu \mathrm{g} / \mathrm{ml})$-treated group $(P<0 \cdot 05$; Fig. 1(b)).

\section{Effects of grape seed procyanidin B2 on the apoptosis of mesangial cells treated with high-dose glucosamine}

Similar results were demonstrated using the TUNEL staining (Fig. 2(a)) and flow cytometry (Fig. 2(b)) methods. There were no differences observed between the control and mannitol-treated groups. GlcN significantly increased the percentage of apoptotic cells compared with the control group $(P<0.05)$. Interestingly, the treatment with GSPB2 protected against $\mathrm{GlcN}$-induced cell apoptosis $(P<0.05)$.

\section{Effects of grape seed procyanidin B2 on oxidative stress in mesangial cells treated with high-dose glucosamine}

As shown in Table 2, the activities of GSH-Px and SOD and the content of MDA in the mannitol-treated and control groups

Table 1. Primer sequences used for real-time PCR

\begin{tabular}{lll}
\hline Genes & Forward primer $\left(5^{\prime}-3^{\prime}\right)$ & \\
\hline NRF-1 & TTACTCTGCTGTGGCTGATGG & Reverse primer $\left(5^{\prime}-3^{\prime}\right)$ \\
TFAM & GAAAGCACAAATCAAGAGGAG & CTGCTTTTCATTGCATGAGATCT \\
mtDNA & ATCCTCCCAGGATTTGGAAT & ACCGGTAGGAATTGCGATAA \\
$\beta$-Actin & TAAAGACCTCTATGCCAACAC & TAAAGCCATGCCAAATGTCTC
\end{tabular}

NRF-1, nuclear respiratory factor 1; TFAM, mitochondrial transcription factor A; mtDNA, mitochondrial DNA. 


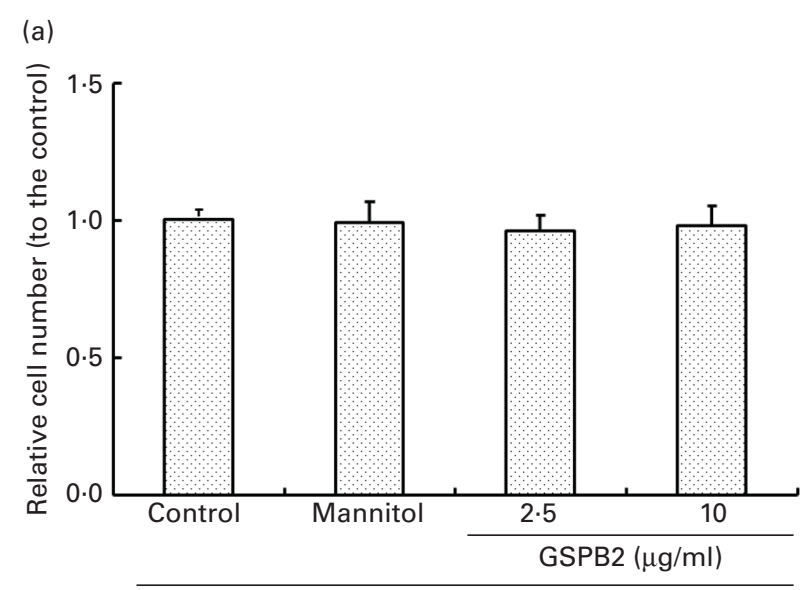

(b)

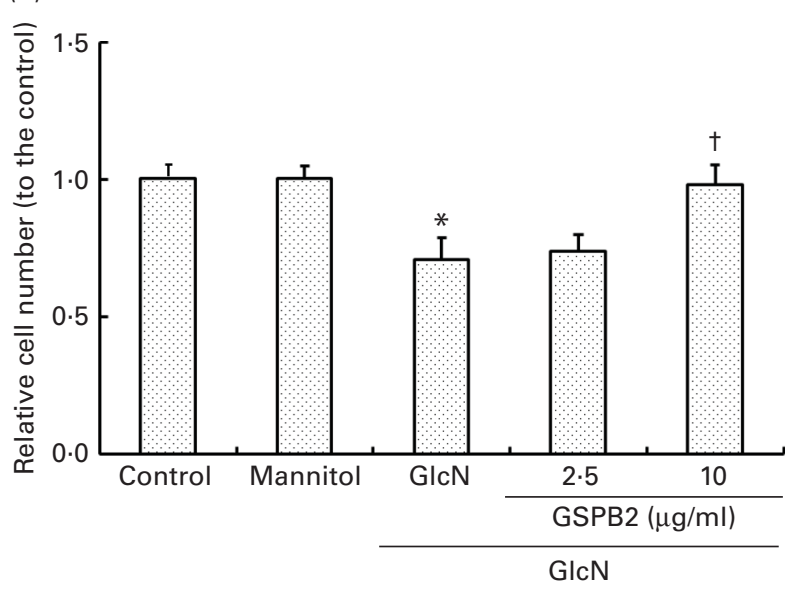

Fig. 1. Effects of grape seed procyanidin B2 (GSPB2) on the viability of mesangial cells cultured under (a) normal glucose (NG) concentrations assessed by the 3-(4,5-dimethylthiazol-2-yl)-2,5-diphenyltetrazolium bromide (MTT) assay and (b) high-dose glucosamine assessed by the MTT assay. Values are means for at least three independent experiments, with standard deviations represented by vertical bars. ${ }^{*}$ Mean value was significantly different from that of the control group $(P<0.05)$. † Mean value was significantly different from that of the glucosamine $(\mathrm{GlcN})$-treated group $(P<0.05)$.

were similar. High-dose GlcN-induced oxidative stress in mesangial cells was demonstrated by the decrease in the activities of GSH-Px and SOD in the GlcN-treated group, but an increase in the content of MDA $(P<0.05$ or $P<0.01)$ compared with the control group. On the contrary, the activities of GSH-Px and SOD were improved in the GSPB2 $(10 \mu \mathrm{g} / \mathrm{ml})$-treated group $(P<0.05$ and $P<0.01$, respectively). Although the content of MDA was slightly decreased in the GSPB2-treated group, it did not differ significantly from the control group.

Effects of grape seed procyanidin B2 on poly(ADP-ribose) polymerase cleavage and the expression of glyceraldehyde 3-phosphate dehydrogenase and receptor for advanced glycation end products in mesangial cells treated with high-dose glucosamine

ROS-induced cellular damage in mesangial cells was evaluated by assaying the protein expression levels of PARP, GAPDH and RAGE, as shown in Fig. 3. There were no differences observed between the control and mannitol-treated groups with respect to these parameters. The ratio of full-length: cleaved PARP and the expression of GAPDH were reduced in the GlcN-treated group compared with the control group, while the expression of RAGE was increased $(P<0.05$ for each). The treatment with GSPB2 (especially the $10 \mu \mathrm{g} / \mathrm{ml}$ dose) reversed these parameters significantly $(P<0.05$ for each), which suggested that GSPB2 could protect against cellular damage, thereby inhibiting apoptosis in mesangial cells treated with high-dose GlcN.

Effects of grape seed proanthocyanidin extracts on the mRNA expression of nuclear respiratory factor 1 , mitochondrial transcription factor $A$ and mitochondrial DNA content in mesangial cells treated with high-dose glucosamine

Real-time PCR analysis showed that the mRNA expression of $N R F-1$ and TFAM, two essential mitochondrial biogenesis factors for gene expression in mammals, was significantly decreased in the GlcN-treated group compared with the control and mannitol-treated groups $(P<0.05$ for each; Fig. 4(a)), and the mtDNA copy number was also significantly reduced $(P<0.05$ for each; Fig. 4(b)). Interestingly, these changes were markedly reversed after the treatment with GSPB2 $(P<0.05$ for each). These findings suggest that mitochondrial dysfunction in mesangial cells treated with high-dose GlcN could be ameliorated by GSPB2 treatment.

Effects of grape seed proanthocyanidin extracts on the AMP-activated protein kinase-silent mating type information regulation 2 homologue 1-PPAR $\gamma$ co-activator $1 \alpha$ axis in mesangial cells treated with high-dose glucosamine

The protein expression of the AMPK-SIRT1-PGC- $1 \alpha$ axis in the samples of each group was detected. As shown in Fig. 5, Western blot analysis revealed no significant differences in the protein expression levels of phospho-AMPK, SIRT1 and PGC$1 \alpha$ between the control and mannitol-treated groups. However, these protein levels were markedly decreased in the GlcNtreated group $(P<0.05$ for each). In contrast, the treatment with GSPB2 at the dose of $10 \mu \mathrm{g} / \mathrm{ml}$ resulted in increased protein expression levels of phospho-AMPK, SIRT1 and PGC-1 $\alpha$ when compared with the GlcN-treated group $(P<0.05$ for each).

\section{Discussion}

To the best of our knowledge, the present study was the first to report that GSPB2 could inhibit high-dose GlcN-induced apoptosis of mesangial cells in vitro. This protective effect induced by GSPB2 could be due in part to the suppression of oxidative stress, the amelioration of mitochondrial dysfunction and the activation of the AMPK-SIRT1-PGC- $1 \alpha$ axis in mesangial cells.

Procyanidin absorption depends on the degree of polymerisation. Deprez et al. ${ }^{(24)}$ showed that procyanidin dimers and trimers are absorbed through the human intestinal cell line Caco-2, but procyanidins with an average polymerisation 
(a)



Control

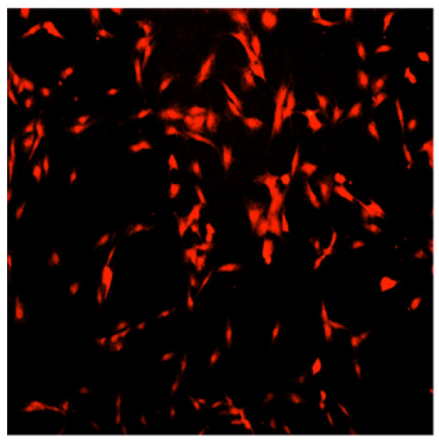

$\mathrm{GlcN}+2 \cdot 5 \mu \mathrm{g} / \mathrm{ml} \mathrm{GSPB} 2$



Mannitol



$\mathrm{GlcN}+10 \mu \mathrm{g} / \mathrm{ml}$ GSPB2

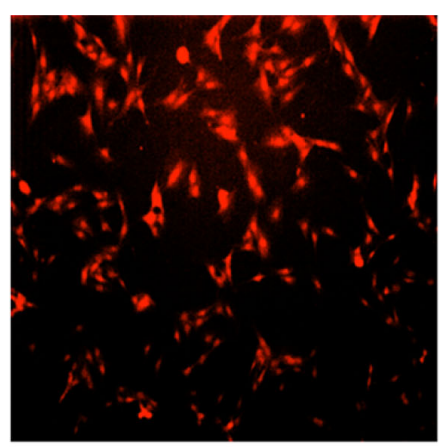

GIcN

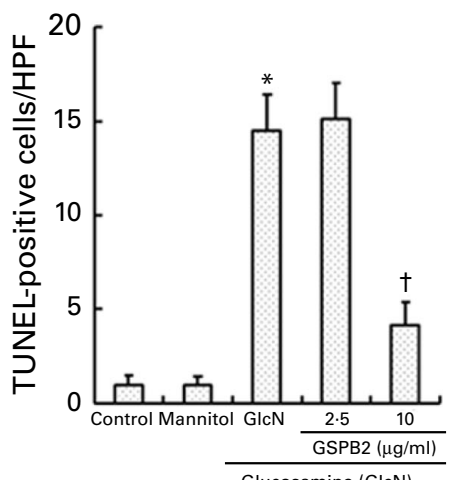

(b)



Control



$\mathrm{GlcN}+2 \cdot 5 \mu \mathrm{g} / \mathrm{ml} \mathrm{GSPB} 2$

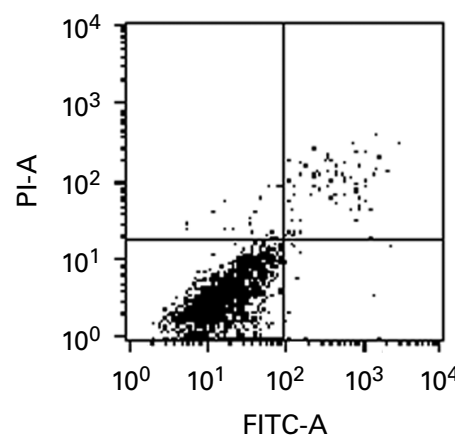

Mannitol

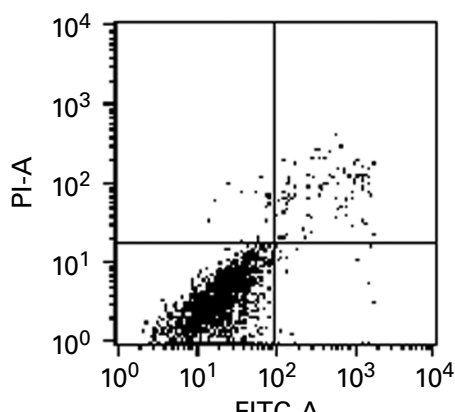

$\mathrm{GlcN}+10 \mu \mathrm{g} / \mathrm{ml}$ GSPB2

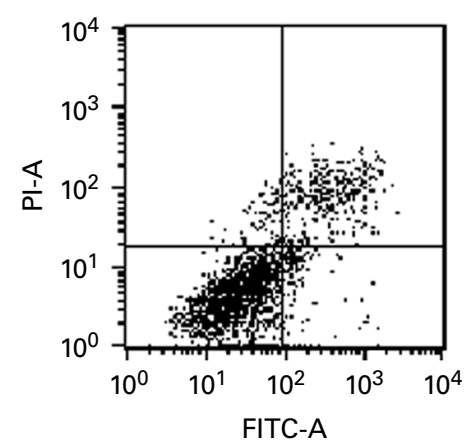

GIcN



Fig. 2. Effects of grape seed procyanidin B2 (GSPB2) on the apoptosis of mesangial cells cultured under high-dose glucosamine. (a) Representative terminal deoxynucleotidyl transferase-mediated dUTP nick-end labelling (TUNEL)-positive mesangial cells (original magnification $\times 400$ ) and the quantitative analyses of the results are shown. HPF, high power field. (b) Annexin V-fluorescein isothiocyanate (FITC-A)/propidium iodide (PI-A) staining of mesangial cells in each group is shown. Values are means from three independent experiments, with standard deviations represented by vertical bars. ${ }^{*}$ Mean value was significantly different from that of the control group $(P<0.05)$. † Mean value was significantly different from that of the glucosamine $(\mathrm{GlcN})$-treated group $(P<0.05)$. (A colour version of this figure can be found online at http://www.journals.cambridge.org/bjn) 
Table 2. Effects of grape seed procyanidin B2 (GSPB2) on glutathione peroxidase (GSH-Px), superoxide dismutase (SOD) and malondialdehyde (MDA) levels in mesangial cells cultured under high-dose glucosamine

(Mean values and standard deviations, $n 6$ per group)

\begin{tabular}{|c|c|c|c|c|c|c|c|c|c|c|}
\hline \multirow[b]{4}{*}{ Parameters } & & & & & \multicolumn{5}{|c|}{ GlcN } & \\
\hline & \multicolumn{4}{|c|}{ Normal glucose } & & & \multicolumn{4}{|c|}{ GSPB2 $(\mu \mathrm{g} / \mathrm{ml})$} \\
\hline & \multicolumn{2}{|c|}{ Control } & \multicolumn{2}{|c|}{ Mannitol } & \multicolumn{2}{|c|}{ GlcN } & \multicolumn{2}{|c|}{2.5} & \multicolumn{2}{|c|}{10} \\
\hline & Mean & SD & Mean & SD & Mean & SD & Mean & SD & Mean & SD \\
\hline GSH-Px (units/mg protein) & 11.95 & 0.32 & 11.77 & 0.43 & $9 \cdot 22^{*}$ & 0.55 & $9 \cdot 37$ & 0.92 & $11.05 \dagger$ & 1.02 \\
\hline SOD (units/mg protein) & $46 \cdot 10$ & 7.05 & $45 \cdot 19$ & 6.98 & $21.91^{\star \star}$ & 3.30 & 30.68 & $5 \cdot 31$ & 41.78†† & $4 \cdot 21$ \\
\hline MDA (nmol/mg protein) & 0.73 & 0.06 & 0.74 & 0.09 & $1 \cdot 17^{\star}$ & 0.29 & 0.89 & 0.23 & 0.71 & 0.26 \\
\hline
\end{tabular}

GlcN, glucosamine.

Mean value was significantly different from that of the control group: ${ }^{*} P<0.05,{ }^{\star \star} P<0.01$

Mean value was significantly different from that of the GlcN-treated group: $† P<0.05$, $\uparrow+P<0.01$.

degree of 7 are unabsorbed. Procyanidin polymers are believed to be fermented by intestinal microflora to produce more smaller molecular-weight phenolic acids and some other unknown metabolites of colonic origin ${ }^{(25)}$. In a study investigating the bioavailability of GSPB2 in plasma, rats were orally administered with procyanidin $\mathrm{B} 2{ }^{(26)}$. The study found that procyanidin B2, epicatechin and 3'-O-methyl-epicatechin were present in the plasma and urine of rats, and procyanidin $\mathrm{B} 2$ reached a maximum plasma concentration at
30-60 min after its consumption. The peak concentration of procyanidin $\mathrm{B} 2$ in plasma was $0.5 \mu \mathrm{M}$ after the administration of procyanidin B2 $(50 \mathrm{mg} / \mathrm{kg}$ body weight). However, further studies are still needed to clarify the mechanisms of the absorption of GSPB2. Additionally, GSPB2 and its metabolites are specifically accumulated in different organs such as liver, adipose tissue and muscle.

A high concentration of glucose $(30 \mathrm{~mm})$ is used to induce the apoptosis of mesangial cells. It has been observed that
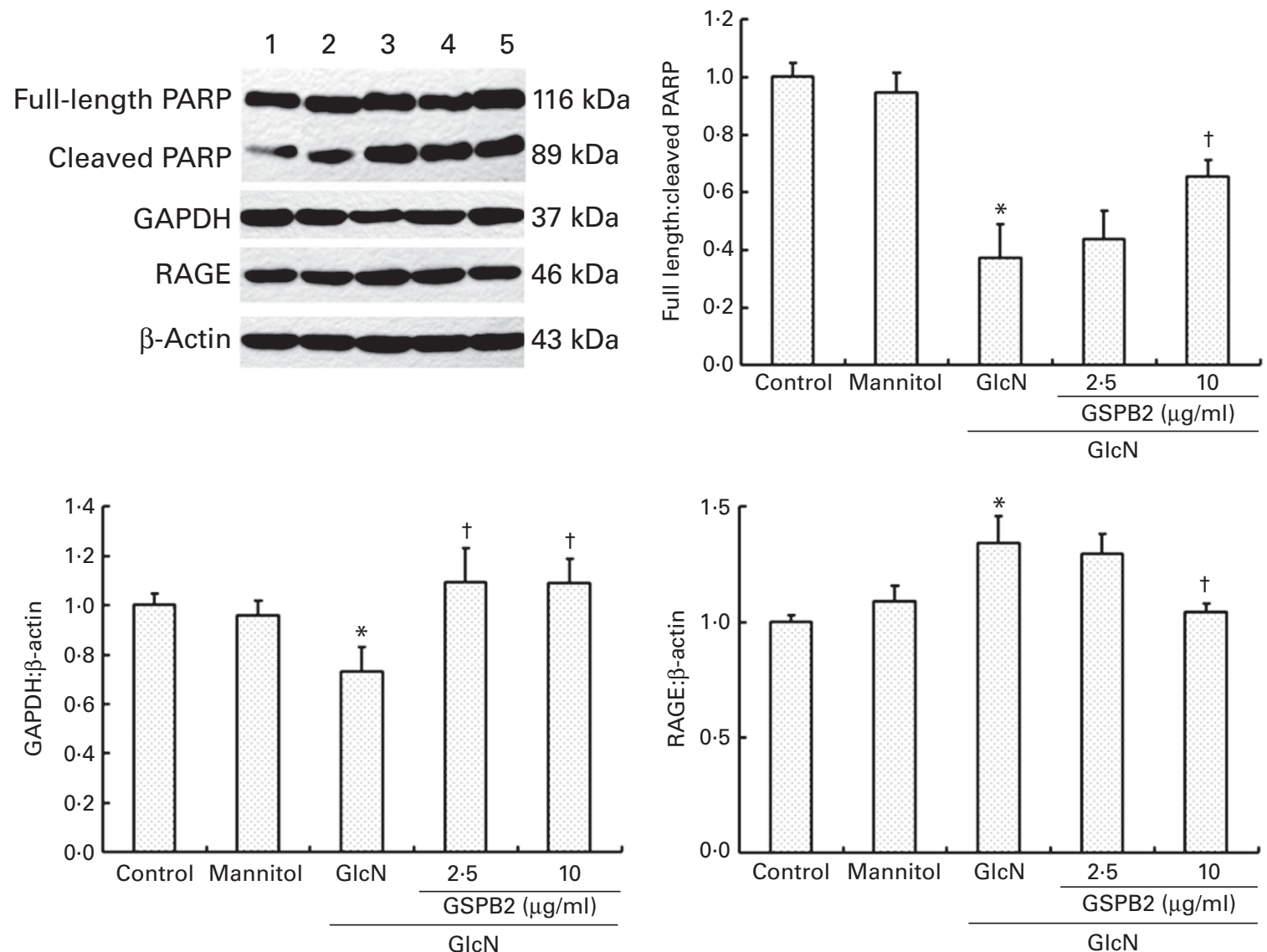

Fig. 3. Effects of grape seed procyanidin B2 (GSPB2) on the protein expression of poly(ADP-ribose) polymerase (PARP), glyceraldehyde 3-phosphate dehydrogenase (GAPDH) and receptor for advanced glycation end products (RAGE) in mesangial cells cultured under high-dose glucosamine. Values were normalised to those of $\beta$-actin as the control. Values are means $(n 4)$, with standard deviations represented by vertical bars. ${ }^{*}$ Mean value was significantly different from that of the control group $(P<0.05)$. † Mean value was significantly different from that of the glucosamine $(\mathrm{GlcN})$-treated group $(P<0.05)$. 

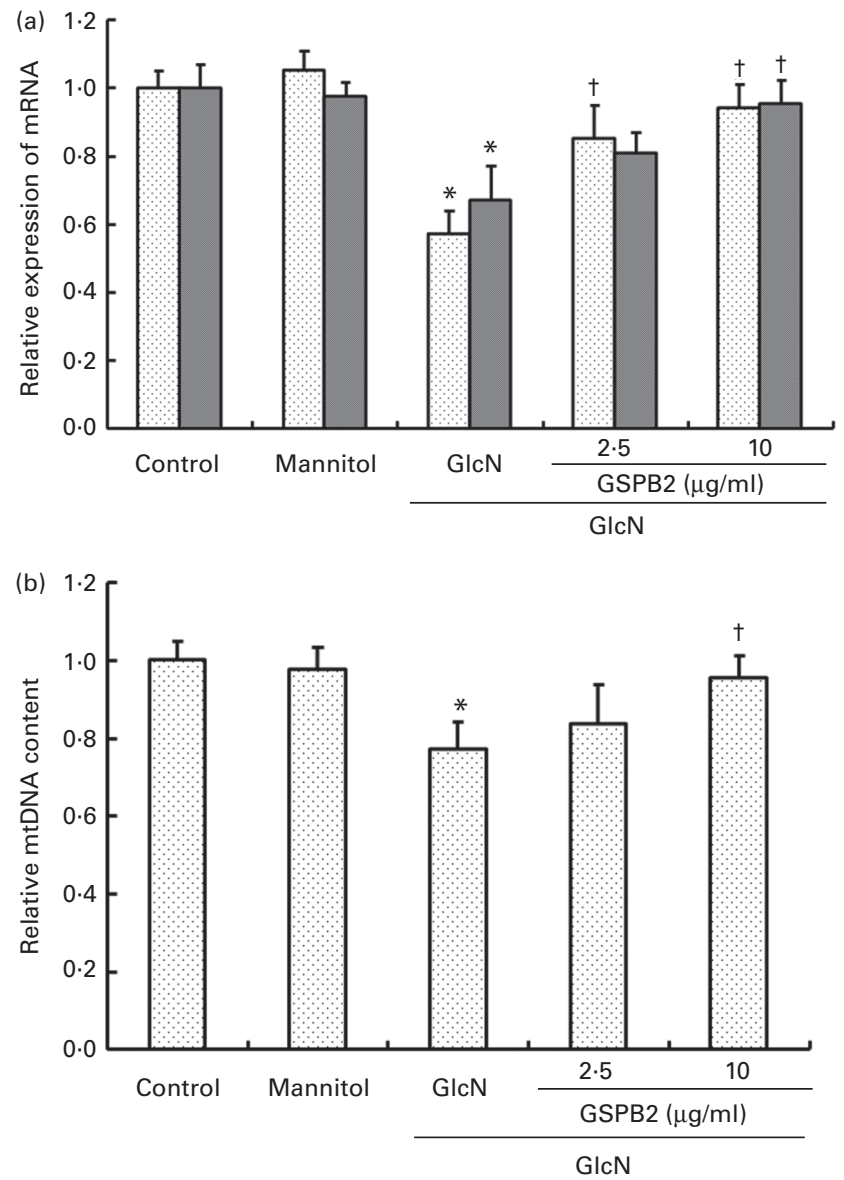

Fig. 4. Effects of grape seed procyanidin B2 (GSPB2) on mitochondrial dysfunction in mesangial cells cultured under high-dose glucosamine as determined by real-time PCR analysis. Effects of GSPB2 on (a) the mRNA expression of nuclear respiratory factor 1 (NRF-1; 圆) and mitochondrial transcription factor A (TFAM; $\square$ ), and (b) mitochondrial DNA (mtDNA) copy number. Values are means $(n 6)$, with standard deviations represented by vertical bars. * Mean value was significantly different from that of the contro group $(P<0.05)$. $†$ Mean value was significantly different from that of the glucosamine $(\mathrm{GlcN})$-treated group $(P<0.05)$

exposure to high levels of GlcN could cause apoptosis as well $^{(27,28)}$. Previous studies have proved that protracted exposure to a high dose of GlcN leads to the apoptosis of mesangial cells in a manner that is similar to that observed in high-glucose conditions ${ }^{(29)}$. In the present study, we observed that mesangial cell apoptosis was induced by the treatment with $15 \mathrm{~mm}-\mathrm{GlcN}$. In addition, since the dose of mannitol, as osmolarity control, was much lower than the high concentration of glucose $(30 \mathrm{~mm})$, the influence of osmotic pressure on the results was minimised. Therefore, the $15 \mathrm{~mm}-\mathrm{GlcN}$ dose was used in the subsequent experiments. As shown previously, GSPB2 could exert protective effects against DN by inhibiting advanced glycation end product-induced apoptosis of endothelial cells ${ }^{(22,23)}$. The present results showed that after the treatment with GSPB2 (especially at a dose of $10 \mu \mathrm{g} / \mathrm{ml}$ ) for $24 \mathrm{~h}$, there was a significant inhibition of mesangial cell apoptosis. These data suggest that GSPB2 plays a role in the prevention of GlcN-induced apoptosis, and GSPB2 therapy can be adopted as an effective approach to resist mesangial cell apoptosis in DN.
Oxidative stress plays a pivotal role in the induction of mesangial cell apoptosis ${ }^{(30)}$. SOD is an enzyme responsible for catalysing the conversion of superoxide anion radical into peroxides, which are then converted into water by GSH-Px, and MDA is the most abundant by-product of lipid peroxidation $^{(31)}$. It has been demonstrated that GSPB2 is a powerful antioxidant, and its antioxidative activity is much stronger than that of vitamins $\mathrm{C}$ and $\mathrm{E}^{(32,33)}$. Consistently, the present results showed that the activities of GSH-Px and SOD were markedly increased after the oral administration of GSPB2. In addition, the production of ROS was increased under the condition of oxidative stress. High levels of ROS induce DNA strand breaks, thereby activating PARP. The enzyme PARP is involved in a number of cellular processes including DNA repair and programmed cell death. During apoptosis, PARP is rapidly cleaved, and thus the detection of cleaved PARP is a diagnostic test for apoptosis in cells ${ }^{(34)}$. Subsequently, the activity of GAPDH that is inhibited by cleaved PARP results in the increased expression of RAGE, which ultimately leads to cellular damage ${ }^{(35)}$. In the present study, we observed ROS-induced cellular damage in mesangial cells treated with high-dose GlcN. However, GSPB2 inhibited the cleavage of PARP, improved the activity of GAPDH and down-regulated the expression of RAGE, which provided more evidence on its protective effects on mesangial cells against cellular damage.

Mitochondrial dysfunction, involved in the pathogenesis of $\mathrm{DN}$, is the terminal event in the process involving oxidative stress-induced programmed cell death in mesangial cells ${ }^{(36)}$. Mitochondria are not only 'the power plant' of the cell, but also the major source of ROS generated as the by-products of oxygen metabolism. mtDNA is a primary target of attack by ROS because it is deficient in DNA repair capacity, lacks histone-like coverage, and is localised close to the electron transport chain ${ }^{(37)}$. ROS may injure mtDNA and impair the electron transport chain, which leads to the production of high levels of $\operatorname{ROS}^{(38)}$. GSPB2 has the capacity to scavenge free radical and reduce the production of ROS due to its powerful antioxidative activity, which provides the possibility for the improvement of mitochondrial dysfunction. To evaluate mitochondrial dysfunction, we selected the following indicators: NRF-1; TFAM; mtDNA copy number. NRF-1 is a positive regulator of TFAM, which plays a role in mitochondrial transcription and regulates the transcription and replication of $\mathrm{mtDNA}^{(39)}$. The mtDNA copy number was identified as a surrogate marker of mitochondrial function ${ }^{(40)}$. Studies have found that PGC- $1 \alpha$, a major regulator of oxidative metabolism and mitochondrial biogenesis, could bind to and co-activate the transcriptional function of NRF-1 on the promoter for TFAM, which, in turn, promotes mtDNA transcription and replication ${ }^{(41,42)}$. The activation of PGC- $1 \alpha$ improves mitochondrial biogenesis following oxidant injury ${ }^{(43)}$ Therefore, PGC- $1 \alpha$ plays a key role in mitochondrial dysfunction and oxidative stress. Although there have been few studies describing the effect of GSPB2 on the expression of PGC- $1 \alpha$, some have showed that polyphenols (e.g. resveratrol) enhance the expression of PGC- $1 \alpha^{(15,44)}$, indicating that GSPB2, which is also one of the polyphenols, might have a 

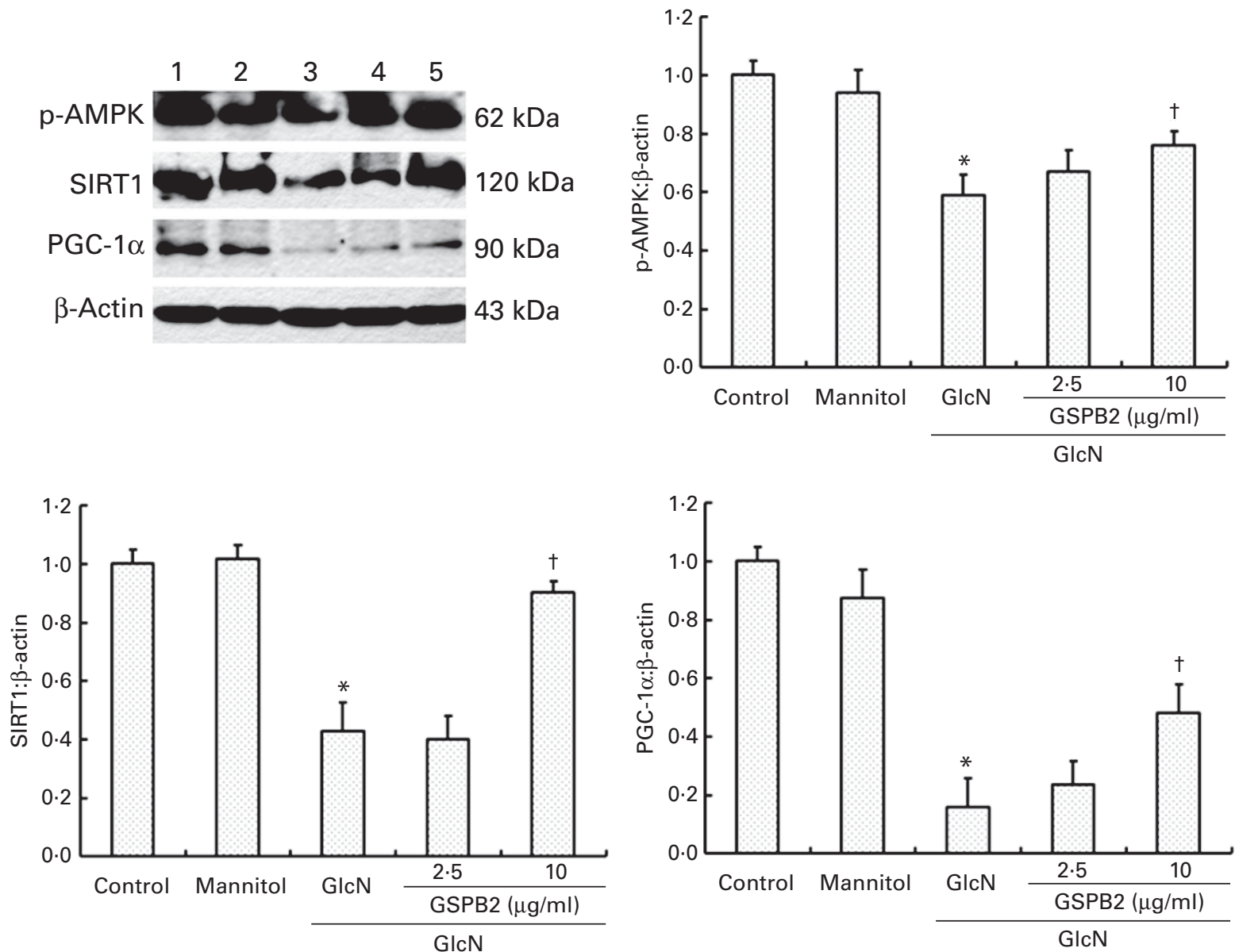

Fig. 5. Effects of grape seed procyanidin B2 (GSPB2) on the protein expression of the phosphorylated AMP-activated protein kinase (p-AMPK)-silent mating type information regulation 2 homologue 1 (SIRT1)-PPAR $\gamma$ co-activator-1 $\alpha$ (PGC-1 $\alpha$ ) axis in mesangial cells cultured under high-dose glucosamine. Values were normalised to those of $\beta$-actin as the control. Values are means $(n 4)$, with standard deviations represented by vertical bars. ${ }^{*}$ Mean value was significantly different from that of the control group $(P<0.05)$. † Mean value was significantly different from that of the glucosamine $(\mathrm{GlcN})$-treated group $(P<0.05)$.

role in promoting the expression of PGC-1 $\alpha$. Moreover, it has been found that GSPB2 activates AMPK and increases the protein levels of SIRT1, which are the regulators of PGC- $1 \alpha$ activity and energy metabolism of mitochondria, in human umbilical vessel cells ${ }^{(45)}$. It has been reported that the activity of the AMPK-SIRT1-PGC-1 $\alpha$ axis is lowered in high glucoseinduced mesangial cells, leading to cell damage, exacerbated oxidative stress as well as apoptotic cell death in diabetes ${ }^{(18)}$. In the present study, the inactivation of the AMPK-SIRT1PGC- $1 \alpha$ axis was found in mesangial cells treated with high-dose GlcN. Interestingly, our data showed that GSPB2 could restore the protein levels of phospho-AMPK, SIRT1 and PGC- $1 \alpha$, suggesting the activation of the AMPK-SIRT1PGC- $1 \alpha$ axis by GSPB2. Furthermore, the present data showed that the activation of the AMPK-SIRT1-PGC- $1 \alpha$ axis increased the expression levels of the transcription factors $N R F-1$ and TFAM, and, subsequently, activated the transcription and replication of mtDNA. These protective effects of GSPB2 on mitochondrial function have also been reported in in vivo studies ${ }^{(41,46)}$

In conclusion, the present study suggested that GSPB2 could inhibit high-dose GlcN-induced mesangial cell apoptosis in vitro, which may be due to the improvement of oxidative stress, mitochondrial dysfunction and the activity of the AMPK-SIRT1-PGC- $1 \alpha$ axis. The AMPK-SIRT1-PGC- $1 \alpha$ axis could be one of the molecular mechanisms involved in the renal protective effects of GSPB2. These results provide further evidence that GSPB2 may be a potential therapeutic agent for the treatment of DN.

\section{Acknowledgements}

The present study was supported by the research grants from the National Natural Science Foundation of China (81102116 and 81072293) and the Research Fund for the Doctoral Program of Higher Education (20110001120026). The funders contributed to the study design, but had no role in the analysis of the study or the writing of this article.

The authors' contributions are as follows: Y. L. and Z. Z. formulated the research question and designed the study; L. B. and X. C. performed the analysis and analysed the data; L. B. wrote the paper; X. C. made some modifications in the manuscript.

The authors have no conflicts of interest to disclose. 


\section{References}

1. Baylis C, Atzpodien EA, Freshour G, et al. (2003) Peroxisome proliferator-activated receptor [gamma] agonist provides superior renal protection versus angiotensin-converting enzyme inhibition in a rat model of type 2 diabetes with obesity. J Pharmacol Exp Ther 307, 854-860.

2. Sheu ML, Ho FM, Chao KF, et al. (2004) Activation of phosphoinositide 3-kinase in response to high glucose leads to regulation of reactive oxygen species-related nuclear factor- $\kappa \mathrm{B}$ activation and cyclooxygenase- 2 expression in mesangial cells. Mol Pharmacol 66, 187-196.

3. Shimizu A, Kitamura H, Masuda Y, et al. (1995) Apoptosis in the repair process of experimental proliferative glomerulonephritis. Kidney Int 47, 114-121.

4. Sugiyama H, Kashihara N, Makino H, et al. (1996) Apoptosis in glomerular sclerosis. Kidney Int 49, 103-111.

5. Ying WZ, Wang PX \& Sanders PW (2000) Induction of apoptosis during development of hypertensive nephrosclerosis. Kidney Int 58, 2007-2017.

6. Mishra R, Emancipator SN, Kern T, et al. (2005) High glucose evokes an intrinsic proapoptotic signaling pathway in mesangial cells. Kidney Int 67, 82-93.

7. Jiao JD, Xu CQ, Yue P, et al. (2006) Volume-sensitive outwardly rectifying chloride channels are involved in oxidative stress-induced apoptosis of mesangial cells. Biochem Biophys Res Commun 340, 277-285.

8. Radeke HH, Meier B, Topley N, et al. (1990) Interleukin 1-alpha and tumor necrosis factor-alpha induce oxygen radical production in mesangial cells. Kidney Int 37, 767-775.

9. Forbes JM, Coughlan MT \& Cooper ME (2008) Oxidative stress as a major culprit in kidney disease in diabetes. Diabetes 57, 1446-1454.

10. Sugiyama H, Kashihara N, Makino H, et al. (1996) Reactive oxygen species induce apoptosis in cultured human mesangial cells. J Am Soc Nephrol 7, 2357-2363.

11. Moreno-Manzano V, Ishikawa Y, Lucio-Cazana J, et al. (2000) Selective involvement of superoxide anion, but not downstream compounds hydrogen peroxide and peroxynitrite, in tumor necrosis factor-alpha-induced apoptosis of rat mesangial cells. J Biol Chem 275, 12684-12691.

12. Santos JH, Hunakova L, Chen Y, et al. (2003) Cell sorting experiments link persistent mitochondrial DNA damage with loss of mitochondrial membrane potential and apoptotic cell death. $J$ Biol Chem 278, 1728-1734.

13. Puigserver P, Wu Z, Park CW, et al. (1998) A cold-inducible coactivator of nuclear receptors linked to adaptive thermogenesis. Cell 92, 829-839.

14. Lin J, Puigserver P, Donovan J, et al. (2002) Peroxisome proliferator-activated receptor gamma coactivator $1 \beta$ (PGC-1 $\beta$ ), a novel PGC-1-related transcription coactivator associated with host cell factor. J Biol Chem 277, 1645-1648.

15. Yuan Y, Huang S, Wang W, et al. (2012) Activation of peroxisome proliferator-activated receptor- $\gamma$ coactivator $1 \alpha$ ameliorates mitochondrial dysfunction and protects podocytes from aldosterone-induced injury. Kidney Int $\mathbf{8 2}$, 771-789.

16. Olmos Y, Valle I, Borniquel S, et al. (2009) Mutual dependence of Foxo3a and PGC-1alpha in the induction of oxidative stress genes. J Biol Chem 284, 14476-14484.

17. Arany Z, Foo SY, Ma Y, et al. (2008) HIF-independent regulation of VEGF and angiogenesis by the transcriptional coactivator PGC-1alpha. Nature 451, 1008-1012.

18. Kim MY, Lim JH, Youn HH, et al. (2013) Resveratrol prevents renal lipotoxicity and inhibits mesangial cell glucotoxicity in a manner dependent on the AMPK-SIRT1-PGC1 $\alpha$ axis in db/db mice. Diabetologia 56, 204-217.

19. Houde V, Grenier D \& Chandad F (2006) Protective effects of grape seed proanthocyanidins against oxidative stress induced by lipopolysaccharides of periodontopathogens. J Periodontol 77, 1371-1379.

20. Li XL, Li BY, Gao HQ, et al. (2009) Effects of grape seed proanthocyanidin extracts on aortic pulse wave velocity in streptozocin induced diabetic rats. Biosci Biotechnol Biochem 73, 1348-1354.

21. Yamakoshi J, Saito M, Kataoka S, et al. (2002) Safety evaluation of proanthocyanidin-rich extract from grape seeds. Food Chem Toxicol 40, 599-607.

22. Li BY, Li XL, Cai Q, et al. (2011) Induction of lactadherin mediates the apoptosis of endothelial cells in response to advanced glycation end products and protective effects of grape seed procyanidin B2 and resveratrol. Apoptosis 16, $732-745$

23. Zhang Z, Li BY, Li XL, et al. (2013) Proteomic analysis of kidney and protective effects of grape seed procyanidin B2 in $\mathrm{db} / \mathrm{db}$ mice indicate MFG-E8 as a key molecule in the development of diabetic nephropathy. Biochim Biophys Acta 1832, 805-816.

24. Deprez S, Brezillon C, Rabot S, et al. (2000) Polymeric proanthocyanidins are catabolized by human colonic microflora into low-molecular-weight phenolic acids. J Nutr 130, 2733-2738.

25. Ferruzzi MG, Lobo JK, Janle EM, et al. (2009) Bioavailability of gallic acid and catechins from grape seed polyphenol extract is improved by repeated dosing in rats: implications for treatment in Alzheimer's disease. J Alzheimers Dis 18, $113-124$

26. Baba S, Osakabe N, Natsume M, et al. (2002) Absorption and

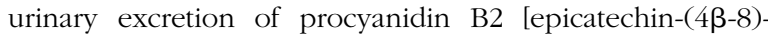
epicatechin] in rats. Free Radic Biol Med 33, 142-148.

27. Fiordaliso F, Leri A, Cesselli D, et al. (2001) Hyperglycemia activates p53 and p53-regulated genes leading to myocyte cell death. Diabetes 50, 2363-2375.

28. Liu K, Paterson AJ, Chin E, et al. (2000) Glucose stimulates protein modification by O-linked GlcNAc in pancreatic beta cells: linkage of O-linked GlcNAc to beta cell death. Proc Natl Acad Sci U S A 97, 2820-2825.

29. James LR, Le C \& Scholey JW (2010) Influence of glucosamine on glomerular mesangial cell turnover: implications for hyperglycemia and hexosamine pathway flux. $A m J$ Physiol Endocrinol Metab 298, E210-E211.

30. Baker AJ, Mooney A, Hughes J, et al. (1994) Mesangial cell apoptosis: the major mechanism for resolution of glomerular hypercellularity in experimental mesangial proliferative nephritis. J Clin Invest 94, 2105-2116.

31. Arivazhagan P, Thilakavathy T \& Panneerselvam C (2000) Antioxidant lipoate and tissue antioxidants in aged rats. J Nutr Biochem 11, 122-127.

32. Bagchi D, Ray SD, Bagchi M, et al. (2002) Mechanistic pathways of antioxidant cytoprotection by a novel IH636 grape seed proanthocyanidin extract. Indian J Exp Biol 40, $717-726$

33. Bagchi D, Garg A, Krohn RL, et al. (1997) Oxygen free radical scavenging abilities of vitamins $\mathrm{C}$ and $\mathrm{E}$, and a grape seed proanthocyanidin extract in vitro. Res Commun Mol Pathol Pharmacol 95, 179-189.

34. Zhang H, Wu H, Wang C, et al. (2014) Acute exposure to thimerosal induces antiproliferative properties, apoptosis, and autophagy activation in human Chang conjunctival cells. Graefes Arch Clin Exp Ophthalmol 252, 275-284. 
35. Giacco F \& Brownlee M (2010) Oxidative stress and diabetic complications. Circ Res 107, 1058-1070.

36. Kang BP, Frencher S, Reddy V, et al. (2003) High glucose promotes mesangial cell apoptosis by oxidant-dependent mechanism. Am J Physiol Renal Physiol 284, F455-F466.

37. Bohr VA (2002) Repair of oxidative DNA damage in nuclear and mitochondrial DNA, and some changes with aging in mammalian cells. Free Radic Biol Med 32, 804-812.

38. Al-Kafaji G \& Golbahar J (2013) High glucose-induced oxidative stress increases the copy number of mitochondrial DNA in human mesangial cells. Biomed Res Int 2013, 754946.

39. Parisi MA \& Clayton DA (1991) Similarity of human mitochondrial transcription factor 1 to high mobility group proteins. Science 252, 965-969.

40. Cho YM, Park KS \& Lee HK (2007) Genetic factors related to mitochondrial function and risk of diabetes mellitus. Diabetes Res Clin Pract 77, S172-S177.

41. Karthikeyan K, Sarala Bai BR \& Niranjali Devaraj S (2007) Grape seed proanthocyanidins ameliorates isoproterenol-induced myocardial injury in rats by stabilizing mitochondrial and lysosomal enzymes: an in vivo study. Life Sci 81, 1615-1621.

42. Cheng M, Gao HQ, Xu L, et al. (2007) Cardioprotective effects of grape seed proanthocyanidins extracts in streptozocin induced diabetic rats. J Cardiovasc Pharmacol 50, 503-509.

43. Wu Z, Puigserver P, Andersson U, et al. (1999) Mechanisms controlling mitochondrial biogenesis and respiration through the thermogenic coactivator PGC-1. Cell 98, 115-124.

44. Rasbach KA \& Schnellmann RG (2007) Signaling of mitochondrial biogenesis following oxidant injury. $J$ Biol Chem 282, 2355-2362.

45. Wang S, Moustaid-Moussa N, Chen L, et al. (2014) Novel insights of dietary polyphenols and obesity. $J$ Nutr Biochem 25, $1-18$.

46. Cui X, Liu X, Feng H, et al. (2012) Grape seed proanthocyanidin extracts enhance endothelial nitric oxide synthase expression through 5'-AMP activated protein kinase/Surtuin 1-Krüpple like factor 2 pathway and modulate blood pressure in ouabain induced hypertensive rats. Biol Pharm Bull 35, 2192-2197. 